\section{TEAGHING LIAISON PSYCHIATRY TO MEDICAL STUDENTS}

By Dr Mrahael A. Smipson, Academic Department of Psychiatry, Royal Free Hospital

Consultation-liaison psychiatry has emerged as a significant area of special interest within clinical psychiatry in recent years. It seeks to enhance the quality of psycho-social care of medically ill patients and of their psychological reactions to illness; to provide effective consultations not only with regard to patient management, but also to the complex problems arising where serious medical and psychiatric illness overlap, and to deal with the personal difficulties arising in members of the clinical team caring for the patient. It has an essential contribution to make to the actual practice of holistic, comprehensive health care; rather than just to the airy rhetoric by which whole-person medicine exists in the medical school's prospectus and objectives but not in its wards. We do not teach mere vapid sympathy, but competent psycho-physiological understanding of the patient, and sound communication skills.

The liaison psychiatrist is especially well placed to teach psychiatry as it applies to the regular, daily, practice of medicine, and in a context which is unusually convincing to those students who do not appreciate the importance of our subject, and who so often show a stubborn tendency to believe of psychiatry only what the surgeon says, if not vice versa.

Examples were provided of the problems encountered in establishing and developing such services, including the nature of the stereotypes of the 'typical' psychiatrist held in other Departments (and often reinforced by our own 'different' behaviour and dress) and the importance of being able to talk in pragmatic and understandable terms, and avoiding unhelpful jargon.

The wide range of clinical problems seen were discussed, with illustrations. The liaison psychiatrist deals with psychiatric illnesses presenting as medical and physical problems, and with medical problems presenting psychiatrically; with the various psychosomatic and stress disorders; deviant sickness behaviour; and with serious, self-destructive noncompliance, excessive dependence, or gross denial of illness. Such difficulties as the unduly angry patient, the seductive patient, the violent or absconding or lying patient, are not adequately discussed in traditional textbooks, or classifiable in traditional nosologies. Problems in the management of the dying patient and his relatives are dealt with in collaboration with the oncologist and haematologist, for example; and while of undeniable importance, often lie beyond the scope of traditional poychiatric therapy.

Far from being an extra super-pecialty, liaison psychiatry represents the most appropriate general psychological medicine to teach the medical undergraduate.

\section{ACUTE PSYGHOTIC REACTIONS IN IMMIGRANTS}

By Dr Roland LrTtuewood, Hackney Hospital and

Dr MAurice LIPSEdge, St Bartholomew's Hospital

Studies of West Indian and West African immigrants have consistently shown high rates of diagnosed schizophrenia, although they differ in the extent to which the illness is seen as atypical. A retrospective examination of hospital notes at an East London Psychiatric Unit showed that although total admissions were similar in different ethnic groups there was an excess of schizophrenia in Caribbean and West African migrants similar to that found previously. These patients also showed an increase in two pathoplastic features, religious and paranoid flavour and an increased proportion of women, formal admissions and short admissions.

A prospective study of patients with religious delusions (a criterion chosen to yield a high proportion of atypical reactions) using the Present State Examination, a Religious Interest Questionnaire, videotapes and interviews with their families, isolated 24 West Indian and West African patients. Sixteen of them who did not have first-rank symptoms of schizophrenia differed from the eight who did in being more likely to be female, to come from a religious family and have had continuous religious experiences acceptable to the family since childhood, to have migrated more than five years before first psychiatric diagnosis/referral, and to have changed church, often to a more charismatic sect, although not participating in church social life. Judged by their family to have been quite well in the week preceding admission, following an event such as a threatened eviction they became excited and violent, assulting people and damaging property, and were taken to hospital under Section 136.

The full PSE could not initially be rated, the patients being suspicious and angry, emotionally labile, often refusing to speak or making delusional misinterpretations. There was no hypomanic affect, thought disorder, clouded consciousness or underlying organic disorder. They complained of auditory 